\title{
Integration of Stamper Fabrication and Design Optimization of LCD Light Guides Using Silicon-based Microfeatures
}

\author{
Jyh-Cheng Yu • Pei-Kai Hsu
}

The original publication is available at www.springerlink.com, http://dx.doi.org/10.1007/s00542-009-0948-5

Received: 29 May 2009 / Accepted: 23 October 2009

Springer-Verlag 2009

\begin{abstract}
This study applies a novel fabrication process that combines anisotropic wet etching of silicon-oninsulator (SOI) wafers with electroforming to manufacture precision stampers. Micron-sized features, such as trapezoidal grooves and truncated pyramidal prisms, can be fabricated and distributed accurately. Because feature geometry and distribution can be accurately realized using the proposed scheme, design optimization of light guide plates (LGPs) becomes realistic. By observing the illumination characteristics of light emitting diode (LED) edge-lit LGPs, the distribution pattern of the LGP is transformed into a parameter design with 7 anchor spacing and the spacing modulation amplitude of the micro features adjacent to LEDs. The proposed fuzzy optimization scheme manipulates distribution parameters to obtain an LGP design with high illumination uniformity. The design of a 3.5-inch LED edge-lit LGP is used as an illustrative example. The optical software program TracePro is applied to simulate luminance performance of BLM. The optimization converges rapidly and provides the optimum design with an average brightness of 2266 (nit) and uniformity of $90 \%$ without use of diffusive sheets. Thus work demonstrates the feasibility and effectiveness of the proposed scheme.
\end{abstract}

\section{Introduction}

Back light modules (BLMs) are major components in thin film transistor liquid crystal displays (TFT-LCDs), which typically consist of light sources, reflector sheets, light guide plates (LGPs), diffuser sheets and prism

\footnotetext{
J.-C. YU* AND P-K HSU

National Kaohsiung First University of Science and Technology,

2, Juoyue Rd., Nantz District, Kaohsiung 811

TAIWAN, ROC.

* Corresponding Author: jcyu@,ccms.nkfust.edu.tw
}

sheets (Fig. 1). Edge light sources, such as cold cathode fluorescent lamps (CCFLs) and light emitting diodes (LEDs), can be converted into uniform planar light by manipulating the design and distribution of the microfeatures on LGP. As the market moves toward reduced thickness and increased brightness of BLMs, edge-lit LEDs and integrated LGPs have become important components in portable display devices.

The size of microfeatures and distribution density directly affect the brightness and uniformity of LGPs. Most LGP designs are patent-protected. Small LGPs often have uniform or a random distribution of microfeatures (Taniguchi and Hira 2000). Reflecting luminous flux is in proportional to the density of microfeatures. Empirical rules suggest that feature density increases as distance from a light source increases. Some researchers have proposed distributing the percentage coverage of a reflecting feature as a power function of the distance from a light source (Fig. 2) (Kashima and Yoshida 1992). Although predetermined mathematical models simplify distribution designs, they may also limit optimization of luminance uniformity. Additionally, a 2D feature distribution scheme must be considered for LED applications. Industrial applications often divide an LGP into small adjacent portions, wherein micro-features are evenly distributed, and apply optimization schemes to search for an optimum density design for each portion (Chang et al. 2007, Sendova 2000).

Manufacturing LGPs uses printed and nonprinted technologies. Printed LGPs are fabricated by printing a diffusive dot matrix onto plain polymer plates. Conversely, nonprinted LGPs are generally injection molded or hot embossed with optical microfeatures integrated into the mold design. Nonprinted LGPs often provide better illumination performance than printed LGPs. The possibility of integrating prism design into 


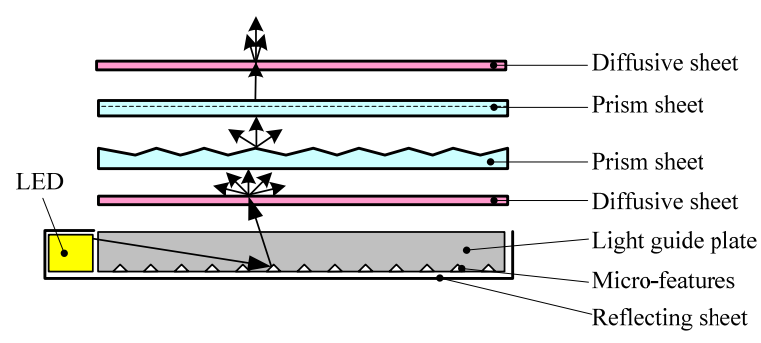

Fig. 1. Schematic diagram of an edge-lit back light module.

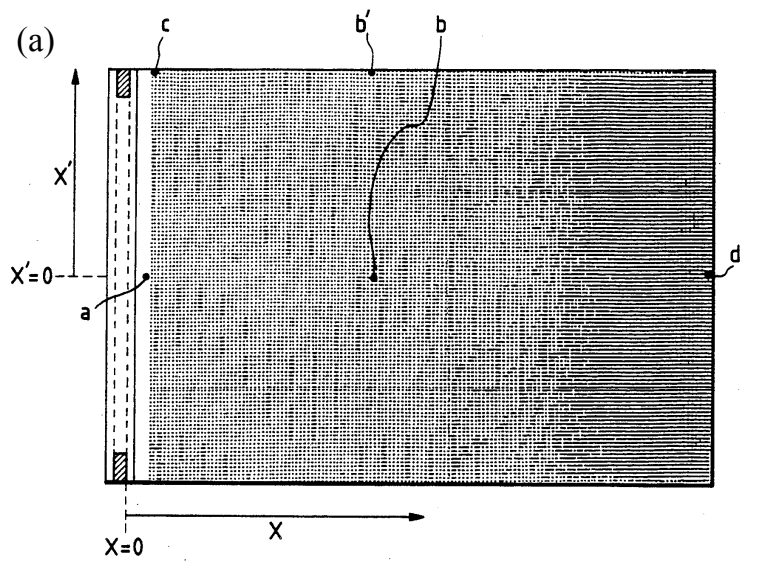

(b)

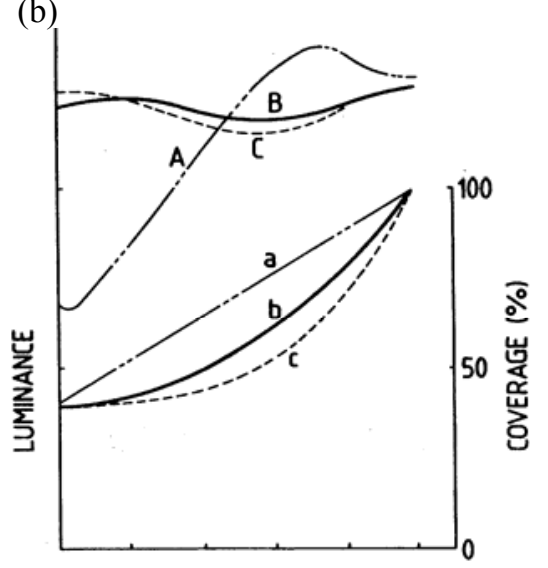

DISTANCE FROM LIGHT SOURCE

Fig. 2. (a) Distribution profile of printed dots of an edge-lit LGP. (b) The percentage coverage of microdots and corresponding luminance distribution vs. distance from a light source.

the emitting side of a nonprinted LGP can also reduce module cost and thickness.

Mold fabrication of nonprinted LGPs is a key technology that can be classified as sandblasting, stamper etching, micro V-cutting, and UV-LIGA. Sandblasting a diffusive pattern and stamper etching are well-developed technologies for mold fabrication. However, the dimension and the accuracy of the microfeatures fabricated using sandblasting and stamper etching are limited, making optical performance unpredictable, requiring considerable mold modifications via trial-and-error. Mold fabrication of V-cut LGPs uses high-speed diamond blade machining to create microgrooves in injection molds. Geometrical accuracy and surface roughness of v-cut on injection molds have been improved markedly. Sophisticated 3D micro features, such as pyramids, can be manufactured. However, the feature distribution is restricted to a simple matrix. Additionally, the requirement of a special machining facility and diamond tool wear are significant cost concerns. Typical techniques for fabricating UVLIGA stampers employ micro-electro-mechanical system (MEMS) technologies to create accurate 2D photo-resist (PR) features using mask designs, and use hightemperature reheating to reform PR patterns into smooth micro-lenses. However, the geometrical curvature of a micro-len is sensitive to PR thickness, surface characteristics, and material properties.

Optical simulation tools, such as TracePro, ASAP, and LightTools, have been widely applied to determine optimum feature distribution for brightness and uniformity of backlights. Geometrical designs of microfeatures for manufacturing are key to closing the gap between optical design and fabrication of LGPs. Design optimization using simulation tools is futile when feature geometry and distribution cannot be realized precisely. Silicon etching and the MEMS technique can be applied to fabricate precision v-slots and pyramid structures for the forming of LGPs and bright enhancement films (BEFs) (Chien and Chen 2006, Lin et al. 2000, Taniguchi et al. 2004). However, the sharp tips of micro features often result in forming and wear problems. Also, the depths of v-slots and pyramids vary with feature widths, becoming a disadvantage when controlling the thickness of LGPs. A good feature design for injection molding should not have any sharp tips.

This study combines anisotropic etching of siliconon-insulator (SOI) wafer and electroforming to fabricate stampers with precision truncated square pyramidal microfeatures. The optimization distribution scheme, which uses iterative fuzzy inference, is applied to generate an LGP design with high brightness and uniformity.

\section{Fabrication of LGP stamper}

Wet chemical etching using anisotropic etchants, such as aqueous potassium hydroxide $(\mathrm{KOH})$ and tetramethylammonium hydroxide (TMAH), can be used to fabricate 3D microfeatures on silicon wafers. The feature geometry after etching is bounded by (111) crystalline planes (Fig. 3). This study controls etching depth by specifying device layer thickness of the $<100>$ SOI wafer to precisely fabricate trapezoidal micro-slots and truncated square pyramids (Yu and Li 2007). A thin 
layer (roughly $1000 \AA$ ) of $\mathrm{SiO}_{2}$ is grown on the SOI via thermal oxidation. The etched wafers are then deposited thin layers of titanium (Ti) and silver (Ag) using E-beam evaporation as the seed layer for electroforming. Nickelbased electroforming is employed to transcribe the silicon features onto a metal plate, followed by backside grinding to desired thickness and flatness. The stamper is finally released using $\mathrm{KOH}$ wet etching. Fig. 4 shows a schematic flowchart of the proposed stamper fabrication scheme.

Two base sizes of truncated pyramids, 10 and $70 \mu \mathrm{m}$, are attempted in this study. The vertex angle of the pyramid is $70.5^{\circ}$ for the etching a $<100>$ wafer. Fig. 5(a) shows a scanning electron microscope (SEM) micrograph of a sample Ni stamper with trapezoidal micro-slots. Fig. 5 (b) shows a stamper with truncated pyramids with base widths of $70 \mu \mathrm{m}$. The trapezoidal cross section of the microfeatures, instead of a sharp tip, can reduce mold wear and increase transcribing accuracy when injection molding an LGP. The proposed stamper fabrication process guarantees extremely high geometrical and dimensional accuracy of the designed injection mold.
Fig. 3. Mask designs and the corresponding features from anisotropic etching $<100>$ wafers. (a)

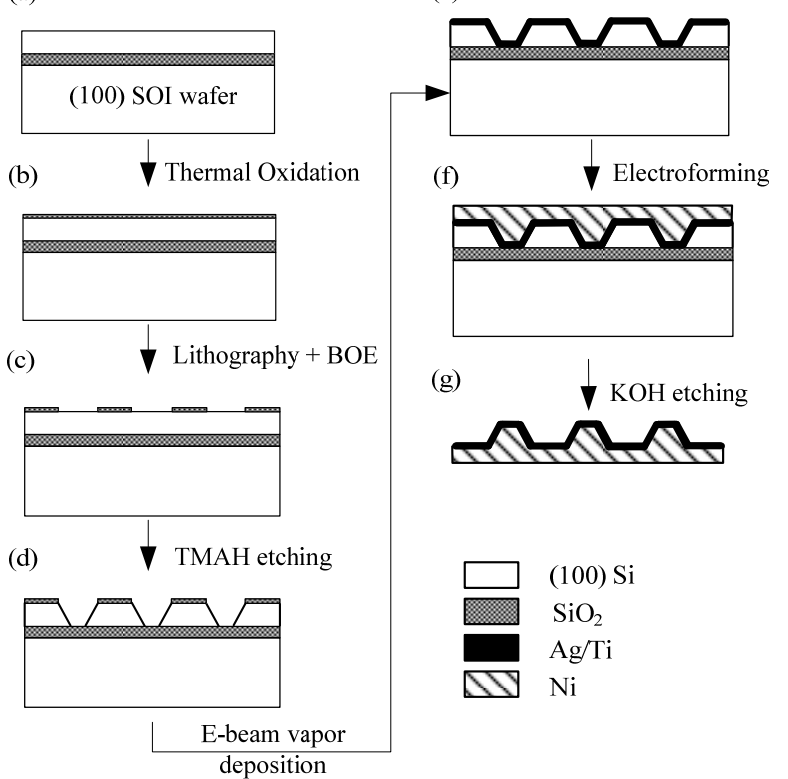

Fig. 4. Schematic flowchart of stamper fabrication. (a)

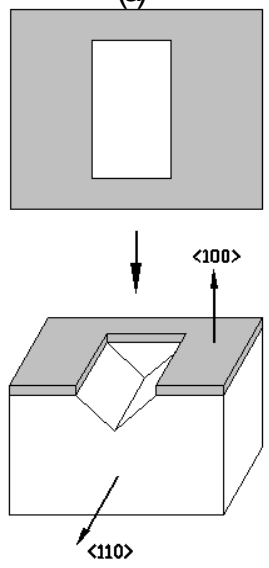

(b)

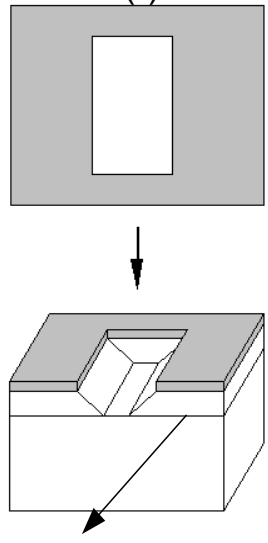

Etch stop layer (c)

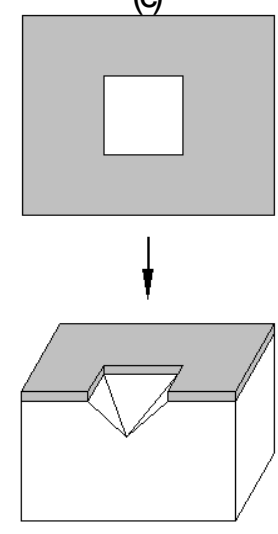

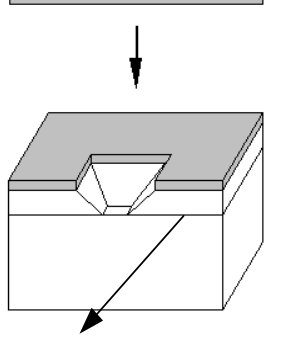

Etch stop layer

(d)

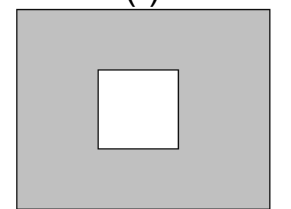

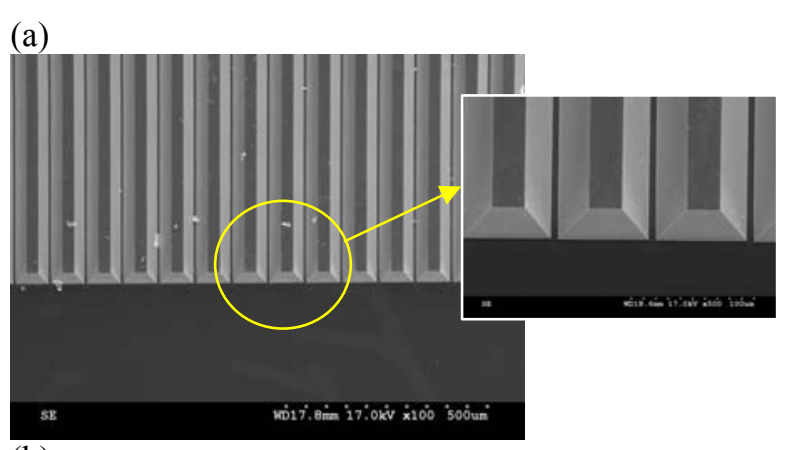

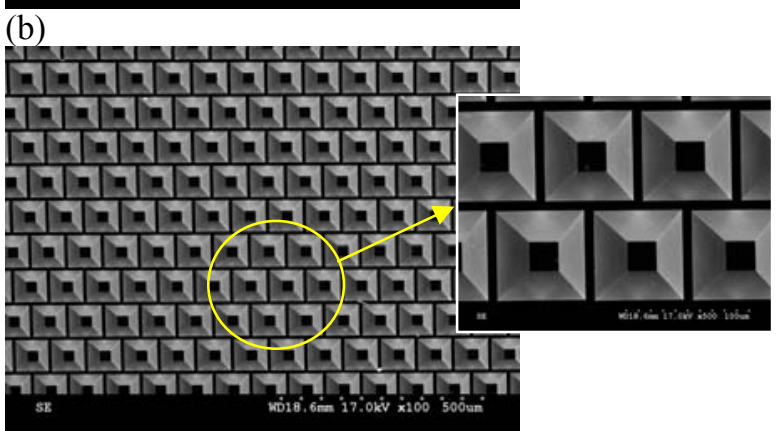

Fig. 5. SEM micrographs of the electroformed stampers from anisotropically etching a $<100>$ SOI wafer. (a) Nickel stamper with trapezoidal microslots. (b) Nickel stamper with truncated square pyramidal microprisms. 


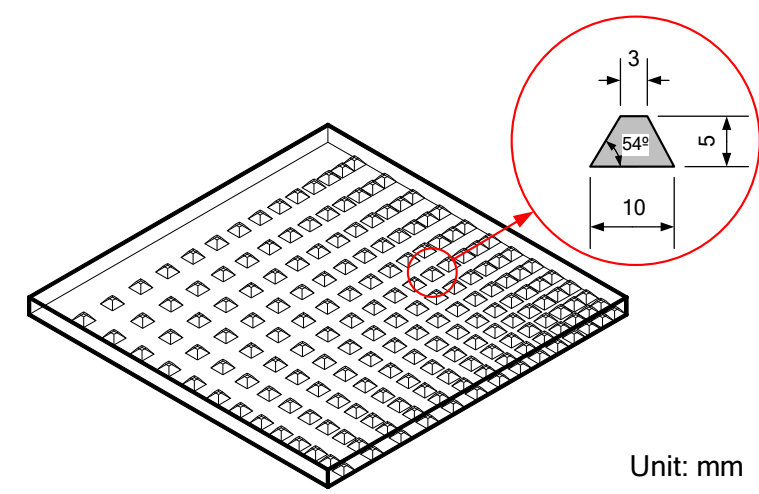

Fig. 6. An LGP with truncated square pyramidal microprisms on its reflecting surface.

\section{Design of LGP}

\subsection{Optical Simulation of BLMs}

The design of a 3.5" LED edge-lit LGP is investigated. An optical ray-tracing simulation tool, TracePro, is applied to simulate the illumination performance of the BLM, which consists of 6 LEDs, 1 LGP, 2 prism sheets of $90^{\circ} \times 50 \mu \mathrm{m}$ and 1 reflecting sheet. The dimensions of the LGP are $73 \times 58 \times 0.76(\mathrm{~mm})$. Six white-light LEDs are arranged on the long side of the LGP. The luminous flux of each LED is $4.238(\mathrm{~lm})$.

The microfeature used in the LGP design is a $10 \times 10$ $\mu \mathrm{m}$ truncated square pyramid from the stamper fabrication process presented in Fig. 4. Fig. 6 shows a schematic distribution and the dimensions of the microfeatures. Consider the transcribing limit of injection molding for a well-controlled geometrical feature. The top of the truncated pyramid is assumed $2 \times 2$ $\mu \mathrm{m}$ by designating the device layer thickness of the SOI to $5 \mu \mathrm{m}$. Because the feature geometry and distribution can be accurately achieved using the proposed stamper fabrication scheme, the optical characteristics of the LGP can be predicted with a high confidence level. Thus, design optimization of the LGP is realistic.

To increase the illumination angle of an LED, prism designs were introduced on the edge of the LGP adjacent to the light source. The prism angle is varied at 70 $130^{\circ}$ and the depth varied at $10-100 \mu \mathrm{m}$ in searching for uniform cross-sectional lighting. Analytical results indicate that a prism angle of $90^{\circ}$ provide the most uniform illumination. Prism depth has no significant effect on lighting distribution and is set at $30 \mu \mathrm{m}$.

Sampled uniformity, such as 9-point uniformity, compares the luminance at several discrete points on the BLM to rapidly assess uniformity, and is commonly used in industry. The average and root mean squared (RMS) error of illuminance across the BLM are used as performance indices because the illuminance distribution of the BLM can be obtained from simulations.

The illumination uniformity of a LGP is influenced by the distribution of microfeatures. Fig. 7 shows an illuminance map obtained from TracePro simulation for an LGP with a uniform distribution of truncated pyramidal prisms. The space between features is $40 \mu \mathrm{m}$. The illuminance distribution along the travelling light is uneven, and the distribution across the light is also uneven, especially for the region close to the LED light sources. The feature distribution must consider illumination uniformity.

\subsection{Distribution Pattern Design}

\subsubsection{Column Spacing}

To simplify the density design, the distribution design of the LGP is first parameterized. The LGP is divided into six sub-regions $\left(R_{1}-R_{6}\right)$ (Fig. 8). The microfeatures are lined up in columns against the light direction. We assume the distribution of column spacing is a function of six continuous line segments (Fig. 9). The distribution function is controlled by seven anchor points. Anchor point $i$ with a column spacing of $s_{i}$ is located at a distance of $x_{i}$ from the light source. The spacing $d$ of the column in region $i$ is determined by linearly interpolating spacing $s_{i}$ and $s_{i+1}$ of two adjacent anchor points. The feature spacing within a column equals column spacing $d$. For instance, the distance $d_{k}$ between column $k$ and the next column in Fig. 10 can be obtained by substituting the distance of column $k$ from the light source into the column spacing distribution function. The feature spacing in column $k$ is also $d_{k}$. Therefore, feature spacing will vary with columns. By manipulating the spacing, $s_{1-}$ $s_{7}$, at anchor points, a uniform illumination distribution in the $\mathrm{X}$ direction can be obtained.

\subsubsection{Modulation of Feature Spacing in the First Region}

Feature spacing within a column is uniform and equal to the column spacing obtained from the column spacing distribution function. Due to the illumination angle of the LED, the illuminance of the portion between LEDs will be lower than the portion in front of the LEDs, especially for the first region, R1, (Fig. 7). Therefore, the feature distribution for the region $R 1$ must vary in the $\mathrm{Y}$ direction to further improve illumination uniformity.

To smooth the illumination distribution in the $\mathrm{Y}$ direction, feature spacing within a column must be adjusted according to the vertical distance from the light source. The feature spacing in front of the light source must be increased and then gradually reduced toward the 

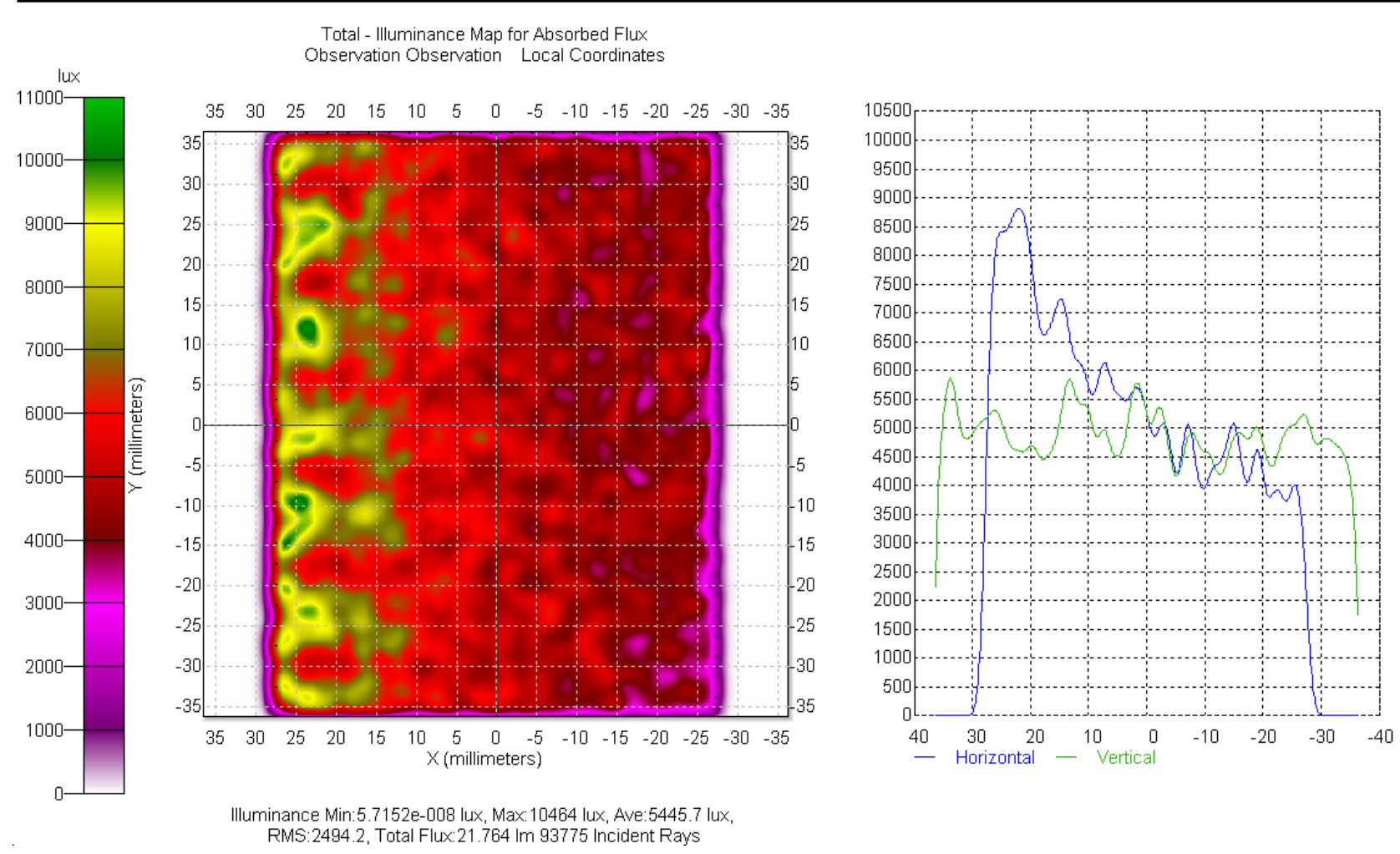

Fig. 7. Illuminance map of an LGP with uniform distribution of truncated square pyramidal microprisms

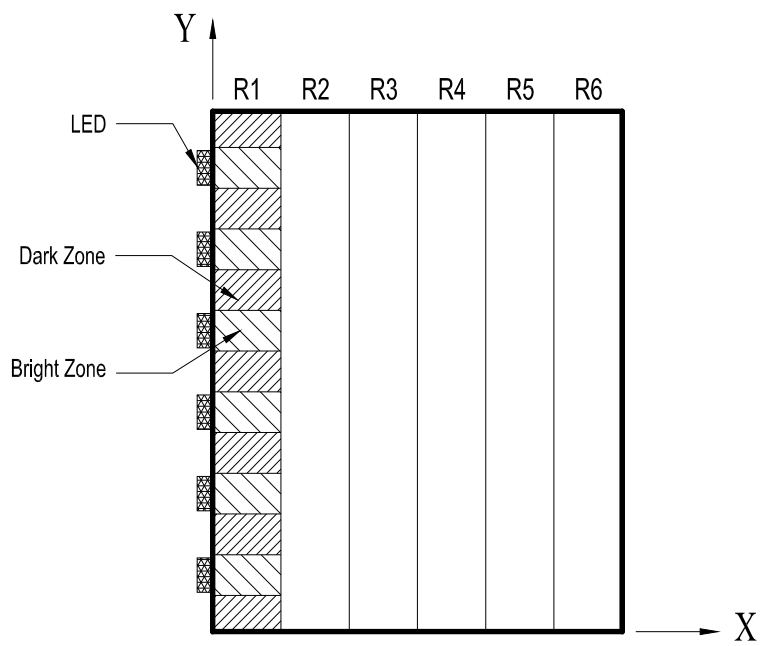

Fig. 8. Division of sub-regions and zones for the pattern distribution of the LGP.

position between the LEDs. The base feature spacing in column $i$ is $d_{i}$, which is obtained from the column spacing distribution function. A sinusoidal function is introduced to modulate feature spacing for columns in $R 1$ using (1).

$$
d_{i j}=d_{i}+a \times \frac{n-(i-1)}{n} \times \sin \left[\frac{2 \pi}{m} \times(j-1)-\frac{\pi}{2}\right]
$$

where $n$ is number of columns in $R 1, d_{i}$ the space between columns $i$ and $i+1, m$ the total number of features between two adjacent LEDs in column $i, j$ the

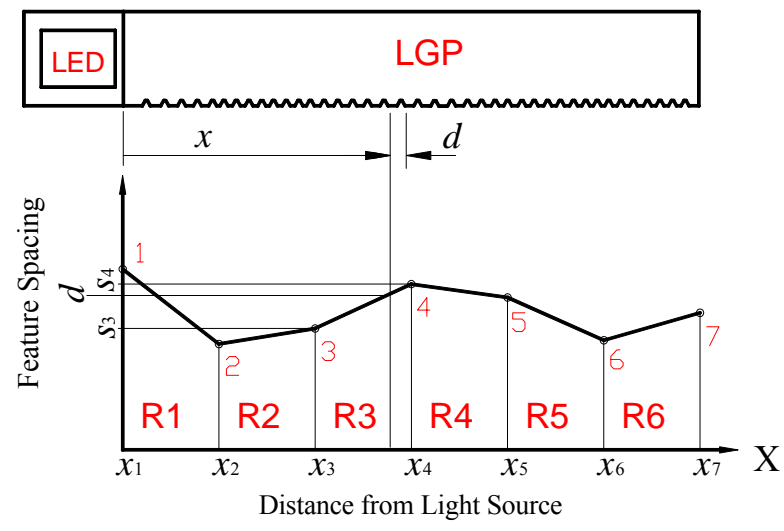

Fig. 9. Column spacing distribution function of the LGP.

feature number $j=1 \sim m$ starting from the symmetrical axis between adjacent LEDs, and $a$ the modulation amplitude for feature spacing in $R 1$.

The number of microfeatures, $m$, in column $i$ between two LEDs can be derived easily by dividing the spacing of LEDs by $d_{i}$. The proposed modulation scheme is symmetrical relative to the center line of an LED. The feature spacing in a column is then modulated from $\left(d_{i}+\right.$ a) for the feature on the center line of an LED to $\left(d_{i}-a\right)$ for the feature at the symmetrical axis between two adjacent LEDs. The feature distribution then becomes fan-shaped (Fig. 10). The modulation amplitude decreases linearly to zero as the feature column 
approaches the end of $R 1$. Therefore, the feature spacing of the last column will again become uniform, ensuring a smooth feature distribution between $R 1$ and $R 2$. By manipulating modulation amplitude, $a$, the uneven illuminance due to the illumination angle of the LED can be improved significantly.

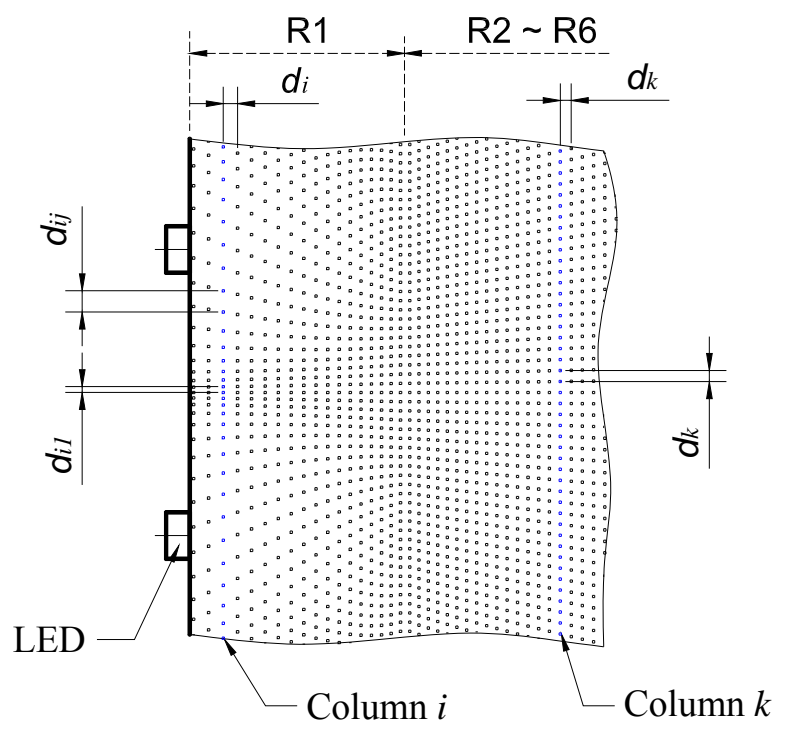

Fig. 10. Modulation of feature spacing for the region closest to the light source

\section{Distribution optimization of microfeatures}

\subsection{Fuzzy Model}

Fuzzy systems are commonly adopted in engineering applications to convert expert decision knowledge into a mathematical reasoning model. Typical fuzzy systems consist of a fuzzifier, a fuzzy rule base, a fuzzy inference engine, and a defuzzifier (Jang et al. 1997). The fuzzifier transforms input data into linguistic fuzzy variables. Expert decision reasoning is then expressed as a set of fuzzy conditional statements based on fuzzy variables. The decision can be reasoned by the fuzzy inference engine; a defuzzifier then converts the linguistic conclusion into a crisp output.

\subsection{Fuzzy Rule Base of the Feature Distribution}

The proposed feature distribution model simplified the LGP design into an 8-variable $\left(s_{1}-s_{7}\right.$, and $\left.a\right)$ optimization problem. Empirical rules suggest increasing feature spacing when a certain region is brighter, and reducing feature spacing when a certain region is darker than average illuminance. However, instead of using the trial-and-error method, the proposed simple optimization scheme, which is based on fuzzy inference, is applied to improve search efficiency. The proposed fuzzy optimization scheme automatically adjusts the feature spacing iteratively until the optimum illuminance uniformity is reached.

The Regional Illuminance Ratio $(R I R)$ is used as a judgment index for adjusting the anchor spacing. The average illuminance of two adjacent regions of an anchor point is compared with average illuminance of the LGP as in (2).

$R I R_{i}=\frac{\text { Average illuminance of adjacent regions at anchor point } i}{\text { Average illuminance of the LGP }}$

If the RIR of anchor point $i$ is greater than one, the corresponding anchor space $s_{i}$ should be increased to reduce the illuminance of adjacent regions. If $R I R_{i}$ is smaller than $1, s_{i}$ should be decreased to increase the illuminance of adjacent regions. Therefore, five singleinput single-output inference rules are proposed based on the empirical decisions as follows:

$R 1$ : If $R I R$ is Too High then Increase the control spacing.

$R 2$ : If RIR is High then Slightly Increase the control spacing.

R3: If RIR is Just Right then Maintain the control spacing.

R4: If RIR is Low then Slightly Decrease the control spacing.

R5: If RIR is Too Low then Decrease the control spacing.

These five levels, Too High, High, Just Right, Low and Too Low, are used to describe the conditioned variables of RIR. Five action levels, Increase, Slightly Increase, Maintain, Slightly Decrease, and Decrease, are defined to describe the Adjustment Factor $(A F)$ for prism spacing at anchor points. Anchor spaces $s_{i}$ will be multiplied by $A F_{i}$ in the next iteration. Fig. 11 and Fig. 12 show the standard membership functions associated with the conditional linguistic and decision variables.

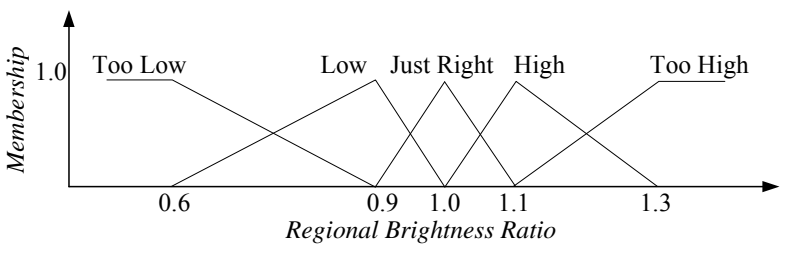

Fig. 11. Membership functions of the regional brightness ratio

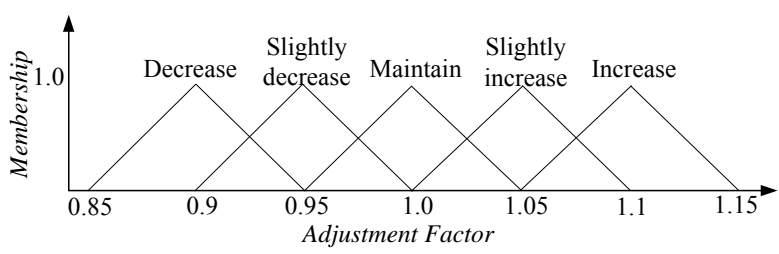

Fig. 12. Membership functions of the spacing adjustment factor 


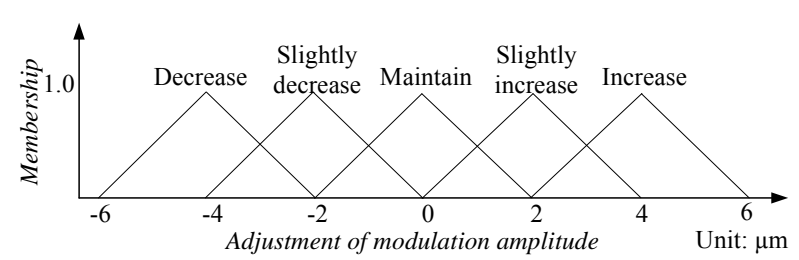

Fig. 13. Membership functions of the adjustment of modulation amplitude

Another set of fuzzy inference rules is employed to adjust the spacing modulation amplitude, $a$, for the first region. The first region is further divided into 13 zones that range from bright to dark zones according to their locations relative to the LEDs. The ratio of average illuminance of bright zones to that of dark zones as in (3) is used as a judgment index for adjusting the spacing modulation amplitude, $a$. If the Zone Illuminance Ratio $(Z I R)$ is greater than one, the modulation amplitude is increased to increase the feature spacing in bright zones, which reduces the illuminance of bright zones and increase the illuminance of dark zones because of the sinusoidal modulation of spacing in (1). Conditioned variables of ZIR adopt the similar levels to that of RIR. Five action levels, Increase, Slightly Increase, Maintain, Slightly Decrease, and Decrease, are defined in Fig. 13 to describe the adjustment of modulation amplitude for the next iteration.

$Z I R=\frac{\text { Average illuminance of bright zones }}{\text { Average illuminance of dark zones }}$

R6: If ZIR is Too High then Increase modulation amplitude.

R7: If ZIR is High then Slightly Increase modulation amplitude.
R8: If ZIR is Just Right then Maintain modulation amplitude.

R9: If ZIR is Low then Slightly Decrease modulation amplitude.

R10:If ZIR is Too Low then Decrease modulation amplitude.

The adjustment factors for seven anchor spaces and the adjusting increment, $\Delta a$, of the modulation amplitude are derived from defuzzification by the inference engine. A simple center average defuzzifier is applied to derive $A F_{i}$ and $\Delta a$ to update the distribution pattern for the next iteration.

\subsection{Design Optimization}

Assume that the initial feature spaces at the seven anchor points are equal. Optical simulation is conducted to acquire the illuminance map for the BLM using the current feature distribution of the LGP. From analysis of the illuminance distribution, fuzzy inference provides a new set of design parameters, $s_{1}-s_{7}$ and $a$, that are used to redesign the distribution pattern of the LGP. The simulation and fuzzy inference iterate until the RMS errors of the illumination distribution converge. Fig. 14 shows the iteration results of average and RMS of the illuminance distribution. The RMS error converges at iteration 24. The corresponding illuminance map for the optimum design (Fig. 15) achieves a significant improvement in illuminance uniformity compared with the uniform feature distribution (Fig. 7). A conventional 9-point uniformity check is measured on the BLM, showing an average brightness of 2266 (nit) and uniformity of $90 \%$ without using any diffusive sheets.

Fig. 14. Iteration result for average and RMS error of illuminance.

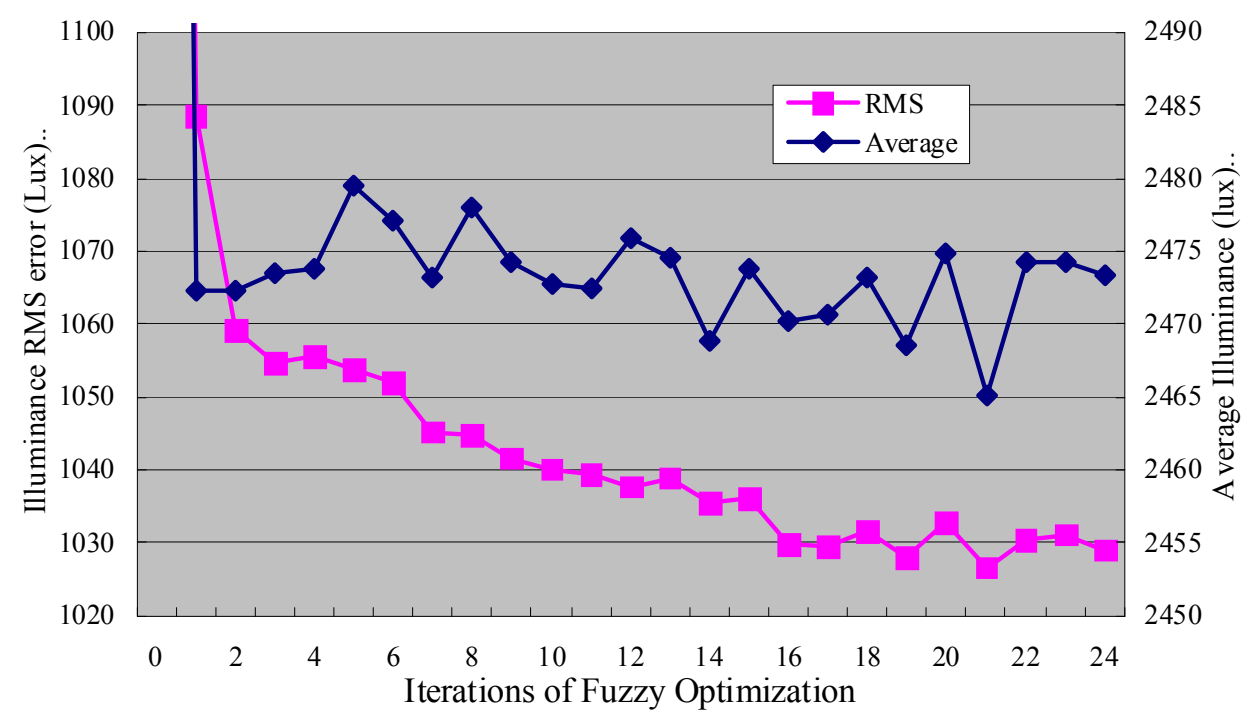



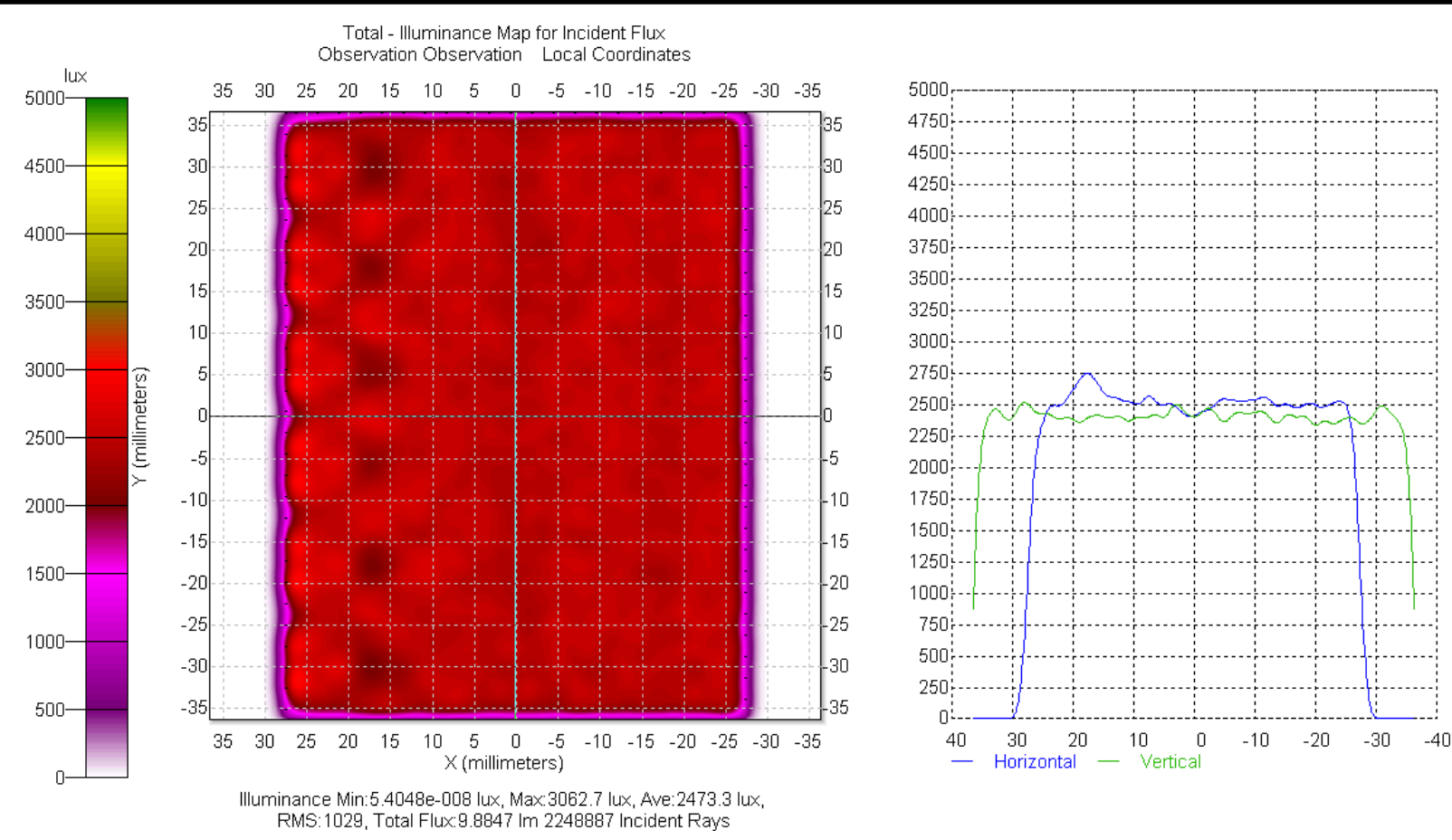

Fig. 15. Illuminance map of the BLM using the LGP with optimum distribution of truncated pyramidal prisms from Fuzzy Optimization

\section{Conclusions}

A precision stamper with silicon-based micron features is fabricated by combining lithography, anisotropic wet etching, and electroforming. This study presents a simple and effective novel optimization scheme to search for the optimum parameter design of the proposed distribution pattern of microfeatures. The RMS error of the illuminance distribution converges smoothly to an optimum parameter design that provides a pattern distribution for the LGP with high illuminance uniformity. The primary merit of this study is the integrated design for manufacturing LGPs. Because feature geometry and the distribution can be accurately realized using the proposed fabrication process, the design optimization of the LGP is realistic. The example design of a 3.5" LED edge-lit LGP demonstrates the feasibility and effectiveness of the proposed scheme.

ACKNOWLEDGMENT This work was supported in part by the Ministry of Economic Affairs of the Republic of China, Taiwan, under Grant 97-EC-17-A-07-S1-108 and the National Science Council under Grant Contract No. 96-2221-E-327-043.

\section{References}

Chang JG, Lee CT, Fang YB, Hwang CC (2007) Generation of random non-overlapping dot patterns for light guides using molecular dynamics simulations with variable r-cut and reflective boundary techniques. Computer Physics Communications 177: $851-862$

Chien CH, Chen ZP (2006) Fabrication of a novel integrated light guiding plate by microelectromechanical systems technique for backlight system. J Microlith Microfab Microsyst 5: 043011

Jang JS, Sun CT, Mizutani E (1997) Neuro-Fuzzy and Soft Computing: a computational approach to learning and machine intelligence. Prentice-Hall

Kashima K, Yoshida N (1992) Back lighting device for a panel. US Patent 5093765

Lin L, Shia TK, Chiu CJ (2000) Silicon-proceed plastic micro pyramids for brightness enhancement applications. J Micromech Microeng 10:395-400

Sendova M (2000) Optimized highly efficient large area modular flat panel display lighting device. US Patent 6132053

Taniguchi H et al. (2004) Method for manufacturing a light guide plate. US Patent 6704070B2

Taniguchi H, Hira Y (2000) Liquid crystal display device. US Patent 6099134

Yu JC, Li PJ (2007) Fabrication of LGP stamper using anisotropic etching of silicon wafers with depth etching stop as the initial electroforming cathode. ROC Patent pending 96100909 